Louisiana State University

LSU Digital Commons

Faculty Publications

Department of Physics \& Astronomy

$1-1-2010$

\title{
SN 2007od: A type IIP supernova with circumstellar interaction
}

\author{
J. E. Andrews \\ Louisiana State University \\ J. S. Gallagher \\ Louisiana State University \\ Geoffrey C. Clayton \\ Louisiana State University \\ B. E.K. Sugerman \\ Goucher College \\ J. P. Chatelain \\ Louisiana State University
}

See next page for additional authors

Follow this and additional works at: https://digitalcommons.Isu.edu/physics_astronomy_pubs

\section{Recommended Citation}

Andrews, J., Gallagher, J., Clayton, G., Sugerman, B., Chatelain, J., Clem, J., Welch, D., Barlow, M., Ercolano, B., Fabbri, J., Wesson, R., \& Meixner, M. (2010). SN 2007od: A type IIP supernova with circumstellar interaction. Astrophysical Journal, 715 (1), 541-549. https://doi.org/10.1088/0004-637X/715/1/541

This Article is brought to you for free and open access by the Department of Physics \& Astronomy at LSU Digital Commons. It has been accepted for inclusion in Faculty Publications by an authorized administrator of LSU Digital Commons. For more information, please contact ir@lsu.edu. 


\section{Authors}

J. E. Andrews, J. S. Gallagher, Geoffrey C. Clayton, B. E.K. Sugerman, J. P. Chatelain, J. Clem, D. L. Welch, M. J. Barlow, B. Ercolano, J. Fabbri, R. Wesson, and M. Meixner 


\title{
SN 2007od: A TYPE IIP SUPERNOVA WITH CIRCUMSTELLAR INTERACTION
}

\author{
J. E. Andrews ${ }^{1}$, J. S. Gallagher ${ }^{1}$, Geoffrey C. Clayton ${ }^{1}$, B. E. K. Sugerman ${ }^{2}$, J. P. Chatelain ${ }^{1}$, J. Clem ${ }^{1}$, D. L. Welch ${ }^{3}$, \\ M. J. Barlow ${ }^{4}$, B. Ercolano ${ }^{4,5}$, J. FABbri ${ }^{4}$, R. Wesson ${ }^{4}$, And M. MeiXner ${ }^{6}$ \\ ${ }^{1}$ Department of Physics and Astronomy, Louisiana State University, 202 Nicholson Hall, Baton Rouge, LA 70803, USA; \\ jandrews@phys.lsu.edu, jgallagher@phys.1su.edu, gclayton@fenway.phys.lsu.edu, jchate6@tigers.1su.edu, jclem@phys.1su.edu \\ 2 Department of Physics and Astronomy, Goucher College, 1021 Dulaney Valley Rd., Baltimore, MD 21204, USA; ben.sugerman@ goucher.edu \\ ${ }^{3}$ Department of Physics and Astronomy, McMaster University, Hamilton, Ontario, L8S 4M1, Canada; welch@ physics.mcmaster.ca \\ ${ }^{4}$ Department of Physics and Astronomy, University College London, Gower Street, London WC1E 6BT, UK; \\ mjb@star.ucl.ac.uk, jfabbri@star.ucl.ac.uk,rwesson@star.ucl.ac.uk \\ ${ }^{5}$ Institute of Astronomy, University of Cambridge, Madingley Road, Cambridge, CB3 0HA, UK; be@ast.cam.ac.uk \\ ${ }^{6}$ Space Telescope Science Institute, 3700 San Martin Drive, Baltimore, MD 21218, USA; meixner@ stsci.edu \\ Received 2009 August 2; accepted 2010 April 8; published 2010 April 29
}

\begin{abstract}
SN 2007od exhibits characteristics that have rarely been seen in a Type IIP supernova (SN). Optical $V$-band photometry reveals a very steep brightness decline between the plateau and nebular phases of $\sim 4.5$ mag, likely due to SN 2007 od containing a low mass of ${ }^{56} \mathrm{Ni}$. The optical spectra show an evolution from normal Type IIP with broad $\mathrm{H} \alpha$ emission, to a complex, four-component $\mathrm{H} \alpha$ emission profile exhibiting asymmetries caused by dust extinction after day 232. This is similar to the spectral evolution of the Type IIn SN 1998S, although no early-time narrow $\left(\sim 200 \mathrm{~km} \mathrm{~s}^{-1}\right) \mathrm{H} \alpha$ component was present in SN 2007od. In both SNe, the intermediate-width H $\alpha$ emission components are thought to arise in the interaction between the ejecta and its circumstellar medium (CSM). SN 2007od also shows a mid-infrared excess due to new dust. The evolution of the H $\alpha$ profile and the presence of the mid-IR excess provide strong evidence that SN 2007od formed new dust before day 232. Late-time observations reveal a flattening of the visible light curve. This flattening is a strong indication of the presence of a light echo, which likely accounts for much of the broad, underlying $\mathrm{H} \alpha$ component seen at late times. We believe that the multi-peaked $\mathrm{H} \alpha$ emission is consistent with the interaction of the ejecta with a circumstellar ring or torus (for the inner components at $\pm 1500 \mathrm{~km} \mathrm{~s}^{-1}$ ) and a single blob or cloud of circumstellar material out of the plane of the CSM ring (for the outer component at $-5000 \mathrm{~km} \mathrm{~s}^{-1}$ ). The most probable location for the formation of new dust is in the cool dense shell created by the interaction between the expanding ejecta and its CSM. Monte Carlo radiative transfer modeling of the dust emission from SN 2007od implies that up to $\sim 4 \times 10^{-4} M_{\odot}$ of new dust has formed. This is similar to the amounts of dust formed in other core-collapse supernovae such as SNe 1999em, 2004et, and 2006jc.
\end{abstract}

Key words: circumstellar matter - dust, extinction - supernovae: general - supernovae: individual (SN 2007od)

\section{INTRODUCTION}

The discovery of massive amounts of dust in luminous infrared (IR) high- $z$ galaxies, seen less than $1 \mathrm{Gyr}$ after the Big Bang (Bertoldi et al. 2003), challenges our understanding of major dust contributors in the universe. After only $1 \mathrm{Gyr}$, there has not been time for low-mass stars to form and evolve to the asymptotic giant branch, which accounts for about half of the dust production in the Galaxy at the current epoch (Dwek 1998). Therefore, it has been suggested that core-collapse supernovae (CCSNe) may be significant contributors to the dust production in high- $z$ galaxies. Surprisingly, dust mass estimates derived from the observed spectral energy distributions (SEDs) of recent $\mathrm{SNe}$ have been much lower than necessary to account for the dust observed in early-time galaxies. The sample of measured $\mathrm{SNe}$ is small but it appears that a typical Type II SN is producing only $10^{-4}$ to $10^{-5} M_{\odot}$ of dust, well below the $1 M_{\odot}$ needed to account for the large amounts of dust seen in the early universe (Kozasa et al. 1989; Todini \& Ferrara 2001). SN 2004et has been found to have formed only a few $10^{-4} M_{\odot}$ of dust (Kotak et al. 2009). Sugerman et al. (2006) showed that larger masses $\left(10^{-2} M_{\odot}\right)$ of dust can be derived in the case of SN 2003gd if the dust is considered to be clumpy and mostly silicate, but Meikle et al. (2007) questioned these findings and derive smaller dust masses $\left(10^{-4} M_{\odot}\right)$ for SN $2003 \mathrm{gd}$. Finally, Ercolano et al. (2007), using similar methods to Sugerman et al. (2006), did not find that large amounts of dust had condensed in the Si-poor envelope of SN 1987A.

There are three distinct signatures of dust formation in CCSNe. These include a drop in visual brightness due to increased dust extinction accompanied by an infrared (IR) excess, and the appearance of asymmetric emission line profiles caused by the greater attenuation by dust in the ejecta of redshifted emission than blueshifted emission. These signatures usually appear a few hundred days after the explosion and only SN 1987A (Lucy et al. 1989; Wooden et al. 1993), SN 2003gd (Sugerman et al. 2006; Meikle et al. 2007), and SN 2004et (Sahu et al. 2006; Kotak et al. 2009) have shown all three.

A few CCSNe have shown evidence for early dust formation. SN 1998S formed dust sometime between days 140-268 (Leonard et al. 2000), while SN 2006jc showed dust formation signatures between days 50-75 (Mattila et al. 2008; Smith et al. 2008) and SN 2005ip from day 60-170 (Smith et al. 2009). The $\mathrm{SNe}$ which show signs of early dust formation are all of Type IIn or Type $\mathrm{Ib} / \mathrm{c}$, where the ejecta interacts with the circumstellar medium (CSM) of the SN. The dust may form in a cool dense shell (CDS) between the forward and reverse shocks (Chevalier \& Fransson 1994; Pozzo et al. 2004). The formation of dust in the CDS allows for an additional avenue of dust formation not available in normal Type II SNe. The capability to form dust both within the SN ejecta and in the CDS suggests that these interacting systems have potential for forming dust in greater 
Table 1

Observation Summary

\begin{tabular}{lcccrr}
\hline \hline Day & JD & Telescope & Instrument & Exposures & Time/Exposure (s) \\
\hline 57 & 2454455 & SMARTS 1.3m & ANDICAM & 5 & 20 \\
232 & 2454630 & Gemini South & GMOS Spectra & 3 & 900 \\
232 & 2454630 & Gemini South & GMOS Imaging & 3 & 20 \\
300 & 2454698 & Spitzer & IRAC & 12 & 100 \\
309 & 2454707 & Gemini South & GMOS Spectra & 3 & 900 \\
309 & 2454707 & Gemini South & GMOS Imaging & 1 & 60 \\
348 & 2454746 & Gemini South & GMOS Spectra & 3 & 900 \\
348 & 2454746 & Gemini South & GMOS Imaging & 2 & 60 \\
455 & 2454853 & Spitzer & IRS PUI & 9 & 15 \\
458 & 2454856 & Gemini North & GMOS Spectra & 3 & 900 \\
458 & 2454856 & Gemini North & GMOS Imaging & 1 & 60 \\
461 & 2454859 & Spitzer & IRAC & 12 & 100 \\
471 & 2454869 & Spitzer & MIPS & 16 & 30 \\
659 & 2455057 & HST & ACS/WFC & 4 & 98 \\
667 & 2454865 & Spitzer & IRAC & 12 & 100 \\
672 & 2455070 & Gemini South & GMOS Spectra & 6 & 1800 \\
672 & 2455070 & Gemini South & GMOS Imaging & 2 & 60 \\
699 & 2455097 & Gemini South & GMOS Spectra & 6 & 1800 \\
709 & 2455107 & HST & ACS/WFC & 4 & 98 \\
811 & 2455109 & HST & ACS/WFC & 4 & 98
\end{tabular}

quantities than normal Type II SNe, although to date they have produced dust amounts comparable to normal Type II SNe. In this paper, we present new spectroscopic and photometric data covering 800 days of the evolution of SN 2007od, an unusual Type IIP SN that shows evidence for CSM interaction and earlytime dust formation.

\section{OBSERVATIONS}

SN 2007od is located in UGC 12846 at a distance of $24.5 \mathrm{Mpc}$ (Immler \& Brown 2007). It was discovered on 2007 November 2 with $R \sim 14.4$ mag (Mikuz \& Maticic 2007), and two days later it was confirmed spectroscopically as a normal Type II SN most closely resembling SN $1999 \mathrm{em}$ at 10 days past explosion (Blondin \& Calkins 2007). Based on this, we assume an explosion date of 2007 October 25 (JD 2454398) throughout this paper. The new observations reported in this paper are summarized in Table 1. We have obtained visible spectroscopy and photometry spanning days $57-811$ as well as mid-infrared photometry on days 300,455 , and 667 of SN 2007od.

Optical imaging was obtained in Johnson-Cousins $B V I$ with the SMARTS consortium $1.3 \mathrm{~m}$ telescope at Cerro Tololo InterAmerican Observatory, Chile. All images were pipeline reduced, shifted, and stacked. Imaging and spectra were also obtained with Gemini/GMOS North and South (GS-2008ADD-2, GS-2008B-Q-45, GN-2008B-Q-245, GS-2009B-Q-1). The $g^{\prime} r^{\prime} i^{\prime}$ images were reduced and stacked using the IRAF Gemini package. The instrumental $g^{\prime} r^{\prime} i^{\prime}$ magnitudes were transformed to standard Johnson-Cousins VRI (Welch et al. 2007; Fukugita et al. 1996). For each night, the transformation involved a least-squares fit with a floating zero point. Late-time images were obtained with the Wide Field Camera (WFC) on the Hubble Space Telescope (HST)/Advanced Camera for Surveys (ACS) using the F435W, F606W, and F814W filters. These images were pipeline reduced, including drizzling and cosmic ray removal, and transformations to the Johnson-Cousins $B V I$ system were accomplished using Sirianni et al. (2005). The large bandpass of the F606W filter includes the $6563 \AA \mathrm{H} \alpha$ emission, which is the main source of the late-time luminosity. We believe that this causes the transformed Johnson $V$ magnitude from

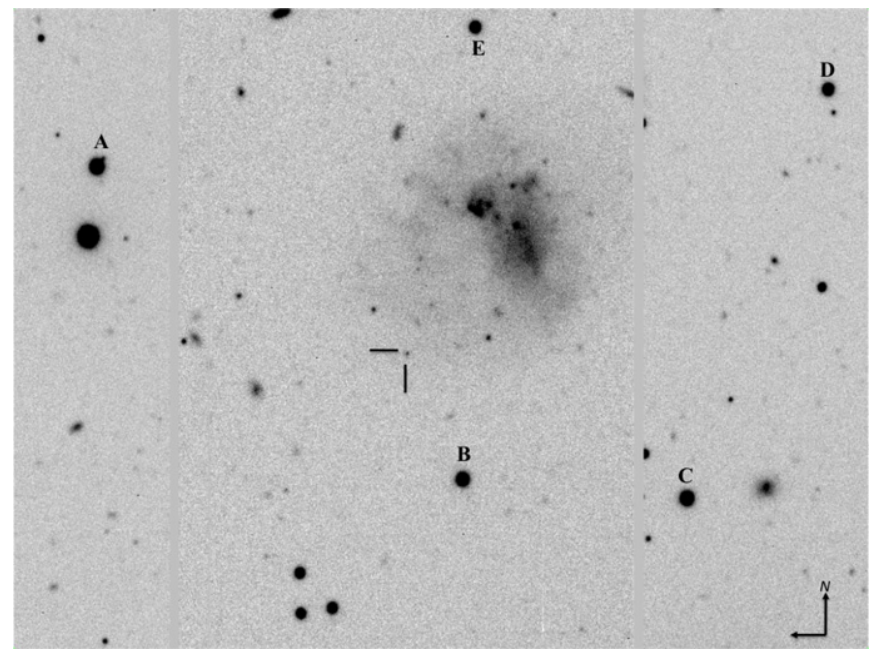

Figure 1. Field of SN 2007od showing the location of the tertiary standards (designated alphabetically) listed in Table 6. The location of SN 2007od is marked. North is up and east is left. The image is $4.7 \times 3^{\prime} .1$ and was taken with Gemini/GMOS-S on 2008 August 29 (day 309) with the $g^{\prime}$ filter.

$H S T$ to appear brighter than the $V$ magnitude obtained from the Gemini transformations. In the Gemini filter set, $r^{\prime}$ contains the $\mathrm{H} \alpha$ flux and is a very small component to the Johnson $V$ transformation but a large component to the $R$ transformation. Therefore, the $R$ magnitude from Gemini would contain the $\mathrm{H} \alpha$ emission whereas $\mathrm{H} \alpha$ would be present in the $V$ magnitude from $H S T$.

A $B V R I$ photometric sequence of tertiary standard stars (as shown in Figure 1) was derived for the SN 2007od field. A table of magnitudes for these standards is located in the Appendix. The VRI light curves of SN 2007od are shown in Figure 2. Uncertainties for the Gemini images were calculated by adding in quadrature the transformation uncertainty quoted in Welch et al. (2007), uncertainty from photon statistics, and the zero point deviation of the standard stars for each epoch. The HST uncertainties consist of the transformation uncertainty from Sirianni et al. (2005) and the uncertainty from photon statistics added in quadrature. We used the narrow Na I D absorption 

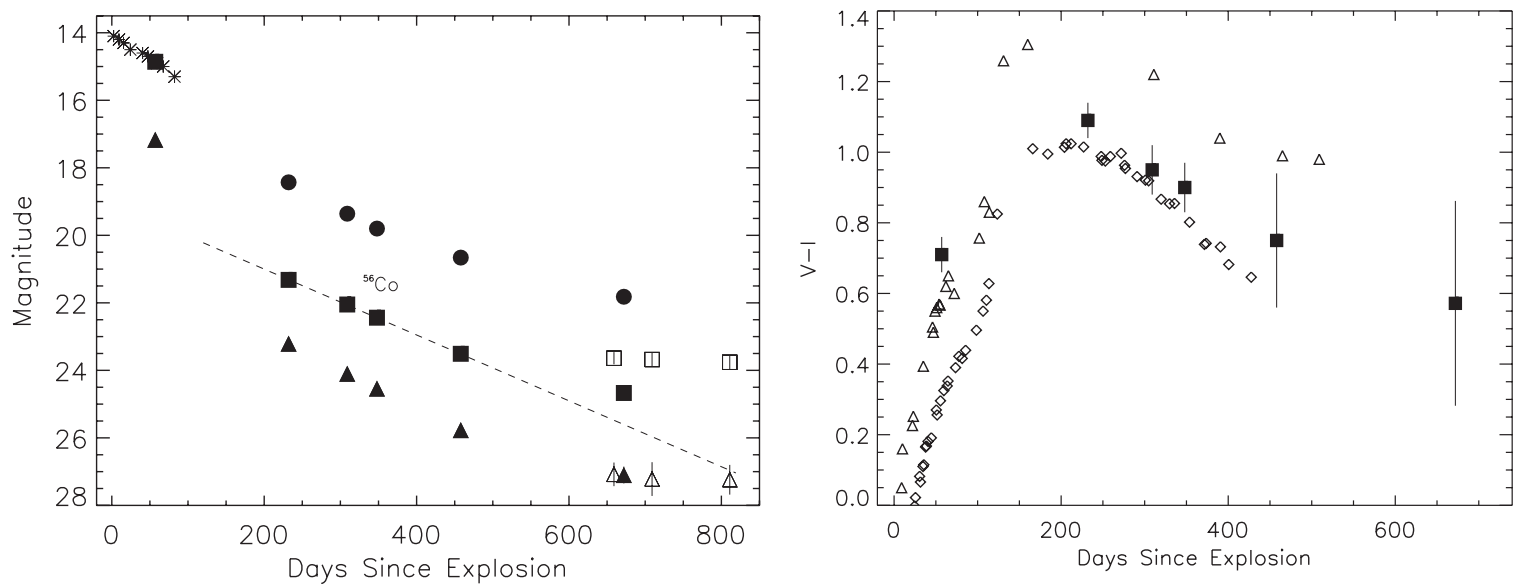

Figure 2. Left: Johnson-Cousins VRI light curves of SN 2007od. $R$ points are shown as circles and have been shifted up 2 mag, $V$ are shown as squares, and $I$ as triangles shifted down $3 \mathrm{mag}$. The filled symbols are from Gemini/GMOS and the open symbols from HST ACS/WFC. Additional photometry of SN 2007od obtained with no filter is plotted (asterisks). The uncertainties are listed in Table 2 and are in most cases smaller than symbol size. The dashed line represents the ${ }^{56} \mathrm{Co}$ decay. Note the drop of $\sim 4.5 \mathrm{mag}$ in $V$ in 20 days, which is large for a Type IIP SN. The flattening of the light curve after day 600 shows the presence of a light echo which is $\sim 10$ mag fainter than the $\mathrm{SN}$ at maximum. The disagreement in $V$ between Gemini and $H S T$ at late epochs is most likely due to filter bandpass differences (see the text). Right: evolution of Gemini $V-I$ of SNe 2007od (filled squares), 1999em (triangles), and 2004et (diamonds; Elmhamdi et al. 2003b; Sahu et al. 2006). The $V-I$ colors of SNe 1999em and 2004et have been corrected for foreground extinction.

Table 2

Optical Photometry of SN 2007od

\begin{tabular}{lccc}
\hline \hline Day & $V$ & $R$ & $I$ \\
\hline 57 & $14.86 \pm 0.04$ & $\ldots$ & $14.17 \pm 0.03$ \\
232 & $21.32 \pm 0.04$ & $20.43 \pm 0.02$ & $20.21 \pm 0.03$ \\
309 & $22.05 \pm 0.05$ & $21.36 \pm 0.03$ & $21.10 \pm 0.05$ \\
348 & $22.44 \pm 0.05$ & $21.80 \pm 0.03$ & $21.54 \pm 0.05$ \\
458 & $23.51 \pm 0.12$ & $22.66 \pm 0.07$ & $22.77 \pm 0.15$ \\
659 & $23.64 \pm 0.18$ & $\cdots$ & $24.08 \pm 0.35$ \\
672 & $24.67 \pm 0.15$ & $23.82 \pm 0.10$ & $24.10 \pm 0.25$ \\
709 & $23.68 \pm 0.21$ & $\cdots$ & $24.22 \pm 0.50$ \\
811 & $23.76 \pm 0.20$ & $\cdots$ & $24.24 \pm 0.44$ \\
\end{tabular}

Notes. Day 57 is SMARTS/ANDICAM photometry, and days 659, 709, and 811 are $H S T$ /ACS. The remaining epochs are Gemini/GMOS photometry.

seen in the day 10 spectrum of SN 2007od (described below) to estimate the foreground extinction. The equivalent width of the blended Na I D lines is $0.50 \pm 0.05 \AA$. This corresponds to $\mathrm{A}_{v} \sim 0.39 \pm 0.04$ (Barbon et al. 1990). Since the value is small, the photometry presented in Figure 2 and Table 2 has not been foreground corrected.

For the first three Gemini/GMOS South epochs, three integrations of 900s were obtained in long-slit mode using grating B600 and a slit width of 0!'75. For the last two epochs on days 672 and 699, six integrations of 1800 s each were obtained. Central wavelengths of 5950,5970 , and $5990 \AA$ for the first three GMOS-S epochs were chosen to prevent important spectral features from falling on chip gaps, and central wavelengths of $6350,6370,6390 \AA$ were chosen for the last two epochs to also include the [Ca II] $\lambda \lambda 7291,7324$ emission. A $2 \times 2$ binning in the low gain setting was used. The one GMOS-N epoch used the R400 grating with central wavelengths of 5460, 5480, and $5500 \AA$. All other settings were the same as in the early GMOS-S epochs. Spectra were reduced using the IRAF Gemini package. The sky subtraction regions were determined by visual inspection and included 30 pixels on either side of the SN. The spectra were extracted using 20 rows centered on the SN. The spectra from each individual night were averaged and have been corrected for the radial velocity of UGC $12846\left(1734 \mathrm{~km} \mathrm{~s}^{-1}\right)$. The spectra obtained on days 672 and 699 have been combined

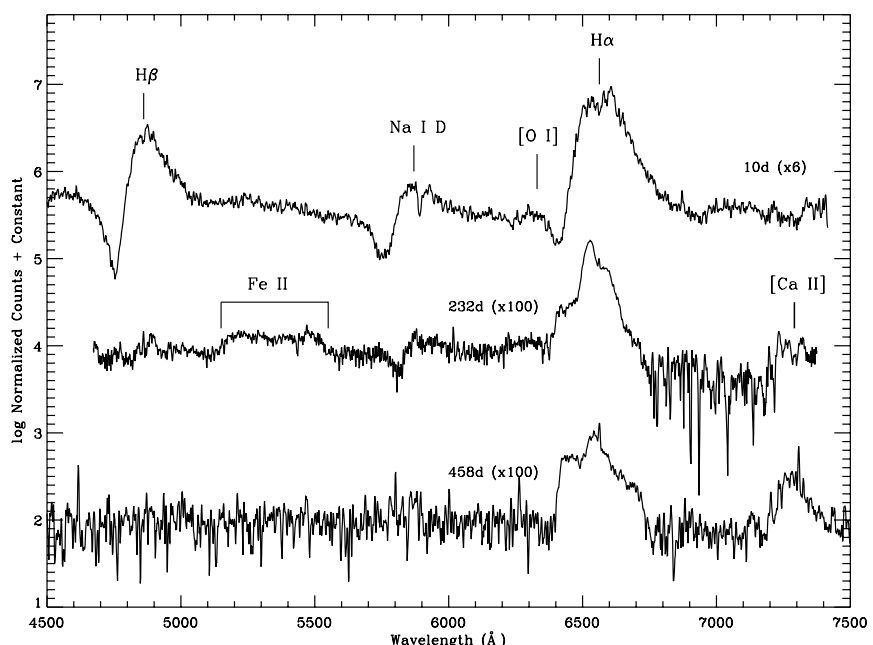

Figure 3. Optical spectra of SN 2007od on days 10, 232, and 458. All spectra have been normalized to the continuum and are shifted vertically by a constant. See the text.

to increase the $\mathrm{S} / \mathrm{N}$. The spectra on days 10 and 50 were taken with the FAST spectrograph operating on the $1.5 \mathrm{~m}$ Tillinghast telescope at the F. L. Whipple Observatory (S. Blondin 2009, private communication). The spectra are shown in Figures 3 and 4.

We obtained three epochs of Spitzer/IRAC imaging and one epoch each with Infrared Spectrograph (IRS) PUI Blue and MIPS. See Table 1 and Table 3. The Spitzer IRAC (3.6, 4.5, 5.8, and $8.0 \mu \mathrm{m})$, IRS PUI Blue $(16 \mu \mathrm{m})$, and MIPS $24 \mu \mathrm{m}$ images were mosaicked and resampled using standard MOPEX procedures to improve photometric quality. Aperture photometry was performed utilizing the prescribed aperture corrections obtainable from the Spitzer Science Center Web site. ${ }^{7}$ Figure 5 presents the SED of SN 2007od at three epochs, Spitzer (day 300)/Gemini (day 309), Spitzer (day 455-471)/ Gemini (day 458), and warm Spitzer (day 667)/Gemini (day 672). The data have not been corrected for foreground

\footnotetext{
7 http://ssc.spitzer.caltech.edu/
} 

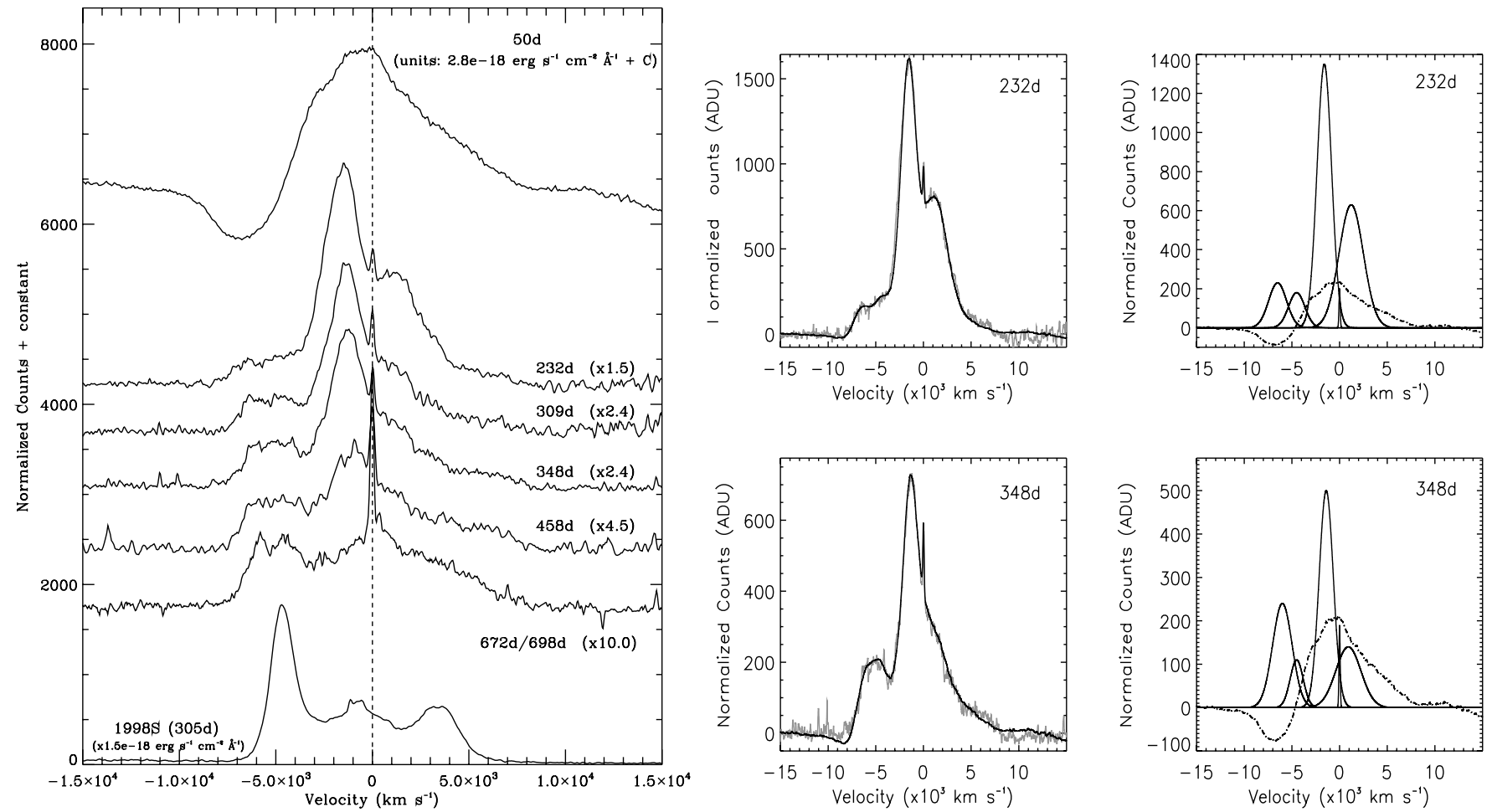

Figure 4. Left: spectral evolution of the $\mathrm{H} \alpha$ profile of SN $2007 \mathrm{od}$. The $-5000 \mathrm{~km} \mathrm{~s}^{-1}$ component is easily visible by day 309 , and the inner $\pm 1500 \mathrm{~km}{ }^{-1}$ components disappear by day 672/699. For comparison, the H $\alpha$ profile of SN 1998S (day 305) has been included (Pozzo et al. 2004). Right: deconvolution of the H $\alpha$ profiles on day 232 and 309 are shown on the right side panels, and the fit is shown as a black line over the spectrum on the left side panels. The dash-dotted line is a scaled day 50 profile, showing that the broad underlying emission at these epochs is consistent with being produced by directly transmitted light from the expanding ejecta. The intermediate width $\pm 1500 \mathrm{~km} \mathrm{~s}^{-1}$ and $-5000 \mathrm{~km} \mathrm{~s}^{-1}$ components are typically produced by CSM interaction as shown by comparison with the SN $1998 \mathrm{~S}$ spectrum. The double Gaussians required to fit the $-5000 \mathrm{~km} \mathrm{~s}^{-1}$ component likely represent density enhancements in the CSM. The day $672 / 699$ spectra, shown on the left, are likely to be mostly due to a light echo which only became a significant contributor when the SN had faded by $\sim 10$ mag.

Table 3

Spitzer Photometry of SN 2007od

\begin{tabular}{lrrcccc}
\hline \hline Day & $3.6 \mu \mathrm{m}$ & $4.5 \mu \mathrm{m}$ & $5.8 \mu \mathrm{m}$ & $8.0 \mu \mathrm{m}$ & $16 \mu \mathrm{m}$ & $24 \mu \mathrm{m}$ \\
\hline 300 & $110.1 \pm 1.6$ & $156.4 \pm 1.8$ & $200.0 \pm 7.6$ & $199.5 \pm 12.2$ & $\ldots$ & $\ldots$ \\
461 & $37.9 \pm 1.4$ & $63.4 \pm 1.6$ & $90.0 \pm 7.5$ & $122.2 \pm 11.8$ & $<59$ & $<35$ \\
667 & $23.5 \pm 0.7$ & $33.3 \pm 0.8$ & $\ldots$ & $\ldots$ & $\ldots$ & $\ldots$ \\
\hline
\end{tabular}

Notes. Fluxes are reported in $\mu \mathrm{Jy}$. The $16 \mu \mathrm{m}$ photometry is from day 455 and the $24 \mu \mathrm{m}$ photometry from day 471 . The day 667 IRAC photometry was obtained with warm Spitzer, which only operates in 3.6 and $4.5 \mu \mathrm{m}$.

extinction. Statistical uncertainties presented in the figure represent $1 \sigma$ errors. It should be noted that the $\mathrm{SN}$ was not detected in the MIPS and IRS/PUI images obtained on days 471 and 455 , respectively. Consequently, these data are upper limits. Table 3 lists the measured mid-IR fluxes of SN 2007od.

\section{DISCUSSION}

\subsection{Light Curve Evolution}

A typical Type IIP SN light curve shows a plateau for the first $\sim 60-100$ days, followed by a sharp drop in luminosity of $\sim 1.5-3$ mag as the $\mathrm{SN}$ transitions into the nebular phase, after which the light curve exhibits an exponential decline powered by ${ }^{56}$ Co decay (Woosley \& Weaver 1986). The light curve of SN 2007od (Figure 2) shows a normal plateau phase followed by an exceptionally large drop in brightness as it is entering the nebular phase. The last photometry before the gap was obtained on day 82 (R. Chornock 2009, private communication). If we assume that the end of the plateau phase took place shortly after that time and we extrapolate the slope of the ${ }^{56}$ Co decay backward into the gap (days 82-232) when no photometry is available, we find that the $V$ brightness fades a minimum of 4.5 mag over the typical 20 day drop period (Elmhamdi et al. 2003a). Woosley \& Weaver (1986), and more recently Kasen \& Woosley (2009), have suggested that if only a small amount of ${ }^{56} \mathrm{Ni}$ is synthesized in the $\mathrm{SN}$ explosion the light curve will show a steep drop between the plateau and nebular phases due to a lack of sufficient thermal energy. Generally, such a steep drop is only seen in low-luminosity $\left(M_{v} \sim-14\right)$ SNe such as $1999 \mathrm{br}, 1999 \mathrm{eu}$, and $2005 \mathrm{cs}$, that have very low inferred ${ }^{56} \mathrm{Ni}$ masses ((2-8) $\times 10^{-3} M_{\odot}$; Pastorello et al. 2004). SN 1994W, which dropped $\sim 3.5 \mathrm{mag}$ from days 110 to 122 (Sollerman et al. 1998), is the only other luminous $\left(M_{v} \sim-18\right)$ Type II besides SN 2007od to show such a steep drop in brightness. Its ${ }^{56} \mathrm{Ni}$ mass was estimated to be $\sim 2.6 \times 10^{-3} M_{\odot}$. Sollerman et al. (1998) suggested that after its 3.5 mag drop from the plateau, SN 1994W declined much faster than the ${ }^{56}$ Co decay once in the nebular phase, possibly due to dust formation.

After day 458, the light curve of SN 2007od shows a deviation from ${ }^{56} \mathrm{Co}$ decay in the form of a flattening of the light curve between days 659 and 811. By day 811, assuming that a constant brightness has been maintained from day 672, the difference in an extrapolated ${ }^{56} \mathrm{Co}$ decay is then $\sim 2.2$ magnitudes in $V$. At late times, the Gemini and HST V magnitudes differ significantly due to differences in the filter bandpasses as discussed in Section 2. However, the constancy of the three epochs of HST/ACS $V$ and I photometry from days 659 to 811 show that the flattening of the light curve is real. It is likely being caused by a light pulse from the SN reflecting off of surrounding material and creating 

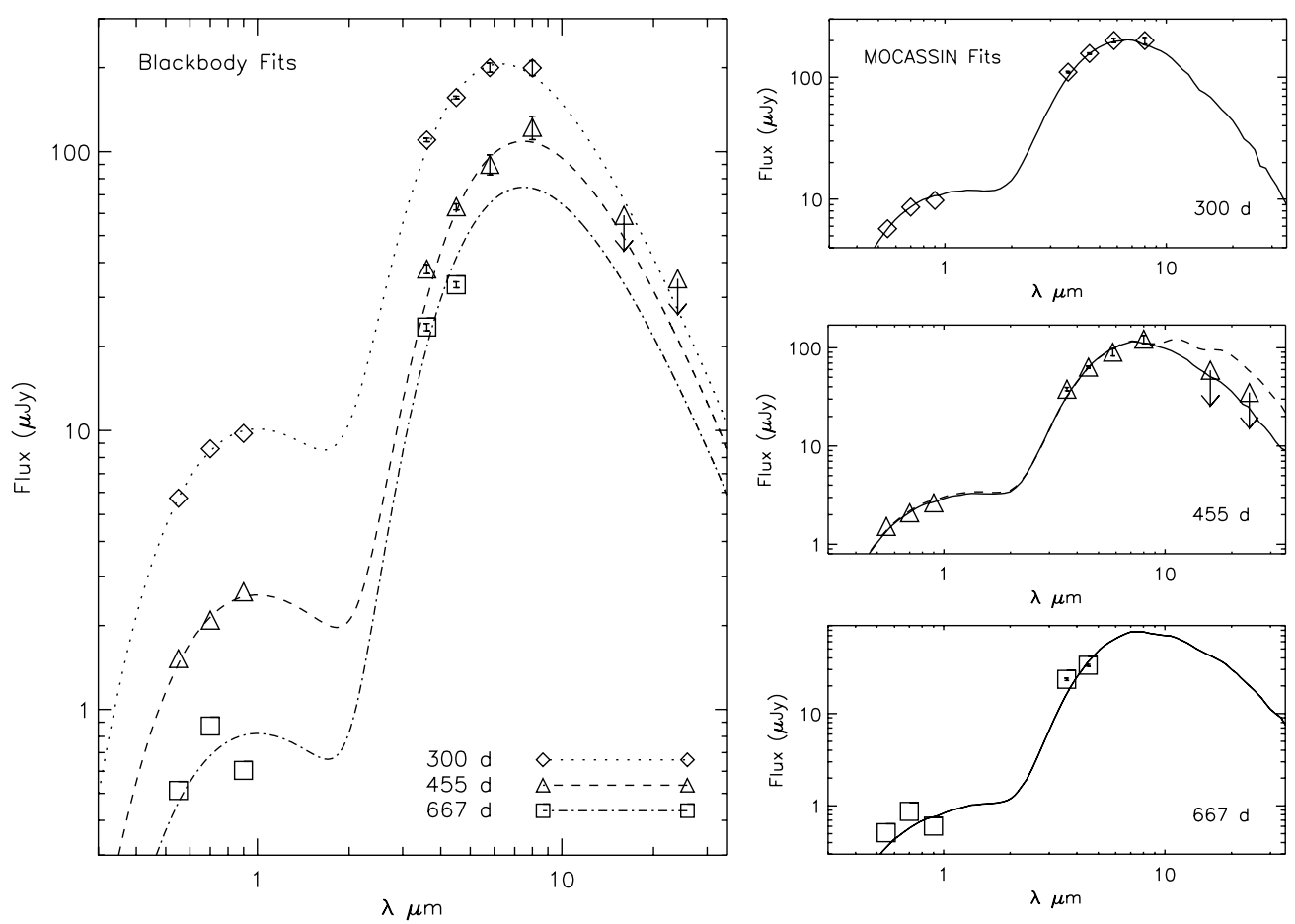

Figure 5. SED analysis of SN 2007od on days $\sim 300$ (diamonds), $\sim 460$ (triangles), and $\sim 667$ (squares). Visible wavelengths were obtained with Gemini/GMOS and IR wavelengths with Spitzer/IRAC, IRS PUI, and MIPS. Dust emission is clearly visible in the 3.6-8 $\mu$ m wavelength range. Left: SEDs with best-fit blackbody curves. The blackbody data are given in Table 4. Optical data were fitted with a simple blackbody while the IR data were fitted with a modified blackbody subject to a $\lambda^{-1}$ emissivity law. Right: the corresponding best-fit MOCASSIN dust models for each epoch. The best fits reflected a smooth distribution of 75\% AC dust (solid lines). For comparison, the best-fit $75 \%$ silicate model for day 455 (dashed line) is shown and predicts too much flux past $10 \mu \mathrm{m}$. MOCASSIN input parameters are shown in full in Table 5 for each model.

a light echo. Given the distance of the SN (24.5 Mpc), platescale of the WFC detector $\left(0^{\prime} .05\right.$ pixel $\left.^{-1}\right)$, and FWHM of the stellar point-spread function (PSF; 1.9 pixel), a source could have a spatial extent on the plane of the sky of up to 0 '! 12 or 46 lt-yr and still be unresolved on the detector. In particular, we find through experimentation that a source with the flux of this SN and with an FWHM up to 1.2 times that of the PSF will not leave detectable residuals when subtracted away, therefore we allow the physical size of the emitting region to be up to 0 '. 12 . Using the light echo equation (Couderc 1939; Sugerman 2003), dust could be up to 475 lt-yr in front of the SN on day 811 and still remains unresolved in the HST imaging. Thus, the echoes could be produced from either circumstellar or interstellar dust. Given that the foreground extinction to the $\mathrm{SN}$ is low $\left(A_{V} \sim 0.4\right)$, any dust producing a light echo should be optically thin. Typically, the contribution from a light echo becomes significant when a SN has faded $\sim 8$ mag from maximum (Patat et al. 2006). SN 2007od was more than 10 mag below maximum light by day 672 when the contribution of the echo to the light curve became significant. A qualitative comparison of the light curve and evolution at late times of SN 2007od to models by Patat (2005) suggests that the echo is more likely due to an interstellar sheet than circumstellar dust. We discuss possible contributions of an echo to the spectral evolution of SN 2007od in Section 3.2.

As shown in Figure 2, the evolution of the $V-I$ colors is normal for a dust-forming Type IIP SN. We have only included the $V-I$ colors from the Gemini observations due to the bandpass considerations of $H S T$ described in Section 2. At the time of dust formation, previous photometry of $\mathrm{SNe} 1999 \mathrm{em}$ and 2004et showed only small $(<0.1 \mathrm{mag})$ changes in their $V-I$ colors. The $V-I$ color evolution of SN 2007od before and after the gap in observations (days 82-232) when we think dust formation began is consistent with these other SNe.
The lack of change in the $V-I$ color evolution indicates that, in general, the color evolution is not sensitive to dust formation. This is likely caused by either a very small amount of dust being formed or larger amounts forming in clumps. If the dust is clumpy, then the color evolution would not reflect the optical depth of dust present and the colors would not be as reddened as they would be if the dust were uniformly distributed, e.g., Witt \& Gordon (1996).

\subsection{Spectral Evolution}

The spectral evolution of SN 2007od shows behavior not previously seen in other SNe. Figure 3 shows the full optical spectrum of SN 2007od at 10, 232, and 458 days. Early-time spectra showed SN 2007od to be a normal Type II SN with a blue continuum, broad $\mathrm{H} \alpha$ emission with a P Cygni profile, and an expansion velocity of $\sim 10000 \mathrm{~km} \mathrm{~s}^{-1}$ (Blondin \& Calkins 2007; R. Chornock 2009, private communication). Other notable spectral features at early times include broad $\mathrm{H} \beta$ emission, weak Na I D $\lambda \lambda 5890,5896$ absorption, and [O I] $\lambda \lambda 6300,6363$ emission that was weaker than that normally seen in Type II SNe. There was no sign of either a narrow (FWHM $\sim 200 \mathrm{~km} \mathrm{~s}^{-1}$ ) H $\alpha$ component or X-ray emission (Blondin \& Calkins 2007; Immler \& Brown 2007), typically seen in a Type IIn SN. If present, the X-ray luminosity of SN 2007od was $<1.0 \times 10^{40} \mathrm{erg} \mathrm{s}^{-1}$, significantly smaller than luminosities measured in Type IIn SNe such as 2006jd and 2007pk (Immler et al. 2007a, 2007b).

By the time of the first Gemini/GMOS epoch (day 232), the spectrum of SN 2007od showed evidence for strong interaction with its CSM, an event usually associated with Type IIn SNe. The $\mathrm{H} \alpha$ emission profile had changed dramatically from the early-time spectra, with the single broad component evolving into at least three intermediate width (FWHM $\sim 1700 \mathrm{~km} \mathrm{~s}^{-1}$ ) 
emission components on top of a fainter, broader emission component (Figure 4). Two of these intermediate width components are arranged symmetrically about zero velocity at $\pm 1500 \mathrm{~km} \mathrm{~s}^{-1}$ and the third, which becomes more prominent at later epochs, lies at $-5000 \mathrm{~km} \mathrm{~s}^{-1}$. We should note that the narrow emission at zero velocity is $\mathrm{H}$ II emission from the host galaxy not associated with the SN. Underlying the three intermediate width components, the broad $\mathrm{H} \alpha$ emission present in the early-time spectra can still be seen, and it is likely that it is responsible for the broad red wing of the $\mathrm{H} \alpha$ emission profile (see Figure 4). Broad, blended Fe II emission between 5100 and $5400 \AA$ similar to that seen in SN 1998S (Pozzo et al. 2004) appeared by day 232 , and [Ca II] $\lambda \lambda 7291,7324$ emission became prominent by day 458 (Figure 3).

A similar multi-component structure was seen in the $\mathrm{H} \alpha$ profile of SN 1998S. Unlike SN 2007od, SN 1998S was classified as a Type IIn and showed both broad and narrow $\mathrm{H} \alpha$ emission components at early times due to the expanding ejecta and photoionized CSM material, respectively. The narrow component disappeared before day 108 (Leonard et al. 2000), and sometime before day 250, a triple-peaked $\mathrm{H} \alpha$ emission profile had developed (Gerardy et al. 2000; Figure 4). The three intermediate width components persisted in SN 1998S from approximately day 250 to day 660 , exhibiting a progressive fading of the red and central components with respect to the blue component; a phenomenon that was attributed to dust formation (Gerardy et al. 2000). The timescale for the evolution of the SN 1998S spectrum is similar to that of SN 2007od, which showed a single broad $\mathrm{H} \alpha$ emission component from days 10 to 114 , followed by a period (114-232 days) when the SN was unobservable, and finally the emergence of multiple-component emission by day 232. In SN 2007od, the blueshifted component at $-1500 \mathrm{~km} \mathrm{~s}^{-1}$ is much stronger than its corresponding component at $+1500 \mathrm{~km} \mathrm{~s}^{-1}$. Like SN 1998S, the simplest explanation for this asymmetry is that newly formed dust is extinguishing the redshifted, far-side emission more than the blueshifted, near-side emission. The ratios of the inner peak heights would require an optical depth of approximately 1.0 at $\mathrm{H} \alpha$ for the asymmetry to be attributable to dust attenuation. If the dust existed in the CSM before the SN explosion, then it would lie outside of the ejecta and all of the emission components would be equally reddened. It is thought that the outer peaks in the $\mathrm{H} \alpha$ profile of $\mathrm{SN} 1998 \mathrm{~S}$ originated in the interaction of the ejecta with a circumstellar ring or torus (Gerardy et al. 2000; Leonard et al. 2000; Pooley et al. 2002; Pozzo et al. 2004; Fransson et al. 2005). If we assume that the same is true of the asymmetric inner peaks of SN 2007od, then there must be dust between the source of the near-side (blueshifted) and the farside (redshifted) emission components in the torus (Figure 6). Moreover, if this dust is forming in the CDS, as was suggested by Pozzo et al. (2004) for SN 1998S, then the emission would likely be coming from a radiative forward shock (Pozzo et al. 2004; Fransson et al. 2005) as one would expect equal attenuation of both redshifted and blueshifted emission originating in the reverse shock (see Figure 6). It is also worthwhile to note that Kotak et al. (2009) have recently alluded to CSM interaction producing a similar multi-component $\mathrm{H} \alpha$ profile in SN 2004et, but occurring at least 400 days after it was seen in SNe 1998S and $2007 \mathrm{od}$.

In $\mathrm{SN} 2007 \mathrm{od}$, the $\pm 1500 \mathrm{~km} \mathrm{~s}^{-1}$ peaks appear sometime between days 114 and 232. Given the maximum velocity of the ejecta of $10000 \mathrm{~km} \mathrm{~s}^{-1}$, this constrains the distance of the inner radius of the CSM responsible for these profile peaks to

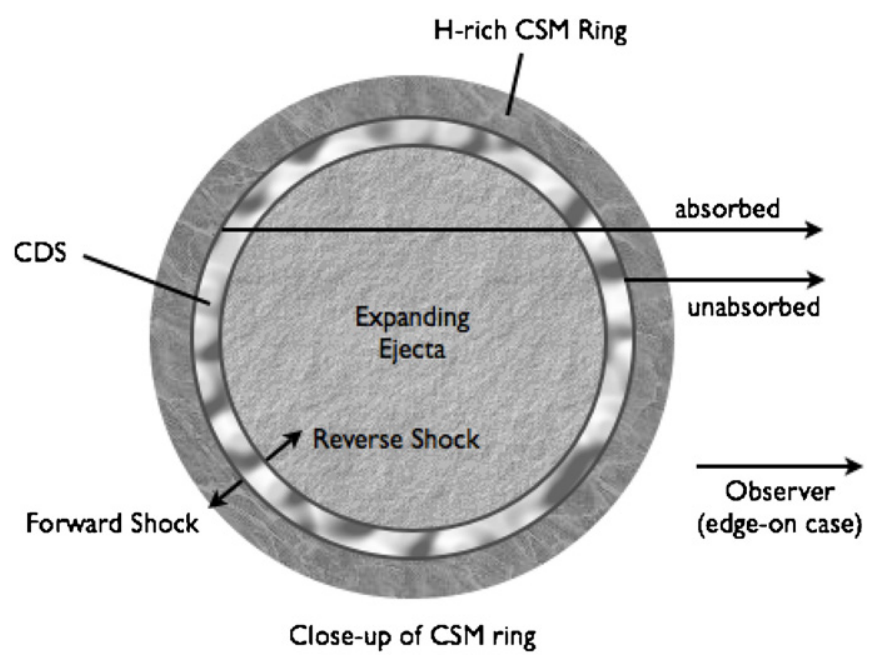

Figure 6. Schematic showing the geometry of SN 2007 od (adapted from Mattila et al. 2008, Smith et al. 2009, and Fox et al. 2009). The inner $\pm 1500 \mathrm{~km} \mathrm{~s}^{-1}$ components may come from the forward shock created when the SN ejecta plowed into a central CSM ring, and the outer $-5000 \mathrm{~km} \mathrm{~s}^{-1}$ components from the ejecta interacting with a blob of CSM out of the plane of the central ring. If the dust is forming in the CDS between the shocks, then the emission coming from the forward shock at the CSM ring explains why the near-side emission is less reddened than the far-side emission. See the text.

700-1300 AU. Assuming a progenitor wind of $10 \mathrm{~km} \mathrm{~s}^{-1}$, this CSM was formed during a mass loss event ending 300-600 yr prior to the SN explosion. With the emergence of the $-5000 \mathrm{~km} \mathrm{~s}^{-1}$ components between days 114 and 309, a similar analysis places this CSM at 700-1700 AU and the formation period between 300 and $800 \mathrm{yr}$ prior to explosion.

The appearance of the asymmetric components between days 114 and 232 implies that dust formation began much earlier than typically seen for non-interacting CCSNe. These SNe (SN 1987A, 2003gd, and 2004et) showed developing asymmetries between days 300 and 600 post explosion due to dust condensation in the ejecta (Lucy et al. 1989; Sugerman et al. 2006; Sahu et al. 2006). Furthermore, the [Ca II] $\lambda 7291$ emission profile in the day 458 spectrum shows only the one broad component, with no intermediate-width components present (Figure 3). This implies that the complex emission seen in $\mathrm{H} \alpha$ is arising in the hydrogen-rich CSM lost from the star before the explosion and not in the metal-enriched ejecta (Gerardy et al. 2000). These observations are more consistent with dust formation in the CDS as has been suggested in SN 1998S (Pozzo et al. 2004; Fransson et al. 2005).

The $\mathrm{H} \alpha$ profile of SN $1998 \mathrm{~S}$ also showed a third peak near zero velocity that has been attributed to the interaction of the ejecta with a spherical distribution of shocked CSM clouds (Gerardy et al. 2000; Leonard et al. 2000; Pooley et al. 2002; Pozzo et al. 2004; Fransson et al. 2005). In the case of SN 2007od, Figure 4 also shows the emergence of a third peak at $-5000 \mathrm{~km} \mathrm{~s}^{-1}$ that was hidden by the broad ejecta emission on day 232. This blue peak becomes increasingly more prominent as the broad underlying ejecta emission begins to fade over time. By day 699, when we believe that the light echo is contributing significantly to the flux, this feature is still prominent. Because a light echo depicts only the early-time emission, it is not possible to explain the $-5000 \mathrm{~km} \mathrm{~s}^{-1}$ peak with a light echo scenario. The nature of this third peak represents the starkest difference between SNe 2007od and 1998S. As previously mentioned, in SN 1998S the peak not associated with the CSM torus was observed at zero velocity and attributed to a spherical 
distribution of shocked CSM clouds. Although a similar solution cannot be invoked to explain a peak centered at $-5000 \mathrm{~km} \mathrm{~s}^{-1}$ as seen in SN 2007od, it is possible that this peak comes from the emission of a single cloud or blob of shocked CSM. If this scenario is correct, because the torus and the blob are seen at different velocities, the blob cannot be located in the plane of the torus. At the same time, the blob would be required to have a velocity component along our line of sight larger than that of the torus. Therefore, if the torus is nearly edge on as viewed from Earth (Figure 6), as hypothesized in SN 1998S, the blob cannot be moving orthogonal to the plane of the torus as this would severely limit its velocity component along our line of sight. On the other hand, if the inclination ${ }^{8}$ of the ring is less than $90^{\circ}$, the blob could be moving orthogonally, but it would require a sufficiently thick torus to retain the dust extinction necessary to explain the asymmetric inner peaks. The scenario presented here to account for the observations is closely based on the scenario used to explain the observations of SN 1998S. This torus + blob scenario is introduced in order to illuminate the manner in which the multi-component $\mathrm{H} \alpha$ emission may arise, but the existing data do not allow the detailed geometry of SN 2007od to be ascertained.

From days 232 to 699 , the relative strength of the emission component at $-5000 \mathrm{~km} \mathrm{~s}^{-1}$ is increasing as the inner pair of emission components $\left( \pm 1500 \mathrm{~km} \mathrm{~s}^{-1}\right)$ slowly disappears. Again if we assume the SN 1998S scenario, then this is explained by the ejecta moving through, and completely destroying, the torus but not the blob. The underlying broad component is also present at all epochs. The broad component is likely a combination of the residual ejecta emission and, given the flattening of our light curve $\sim 10$ mag below maximum, a light echo. The contribution to the total broad component by the light echo is expected to grow with time as the ejecta emission fades. Based on the light curve, on day 238 the light echo flux would be $\sim 20 \times$ fainter than the directly transmitted ejecta emission, and on day 348 the echo would still be $\sim 8 \times$ fainter. However, by day $672 / 699$, the echo flux will likely be greater than the directly transmitted ejecta emission. Therefore, the echo should not be a major contributor to the $\mathrm{H} \alpha$ profile at early times but would be a major contributor of the late-time profile. Figure 4 shows the fits to the day 232 and $348 \mathrm{H} \alpha$ profiles. We have used Gaussians to approximate the intermediate width peaks and a scaled day 50 spectrum to approximate the residual ejecta emission. We should note that the scaled day 50 spectrum is only an approximation to the day 232 and 358 ejecta emission. Realistically, in addition to fading over time, the profile would likely lose its P Cygni absorption, and its overall width would likely decline. The flattopped profile of the $-5000 \mathrm{~km} \mathrm{~s}^{-1}$ component is shown to be well fit by two intermediate width Gaussians possibly due to density enhancements within the CSM cloud or blob. At later times, the profile becomes too complex to fit. On days 458 and $672 / 698$, the $-5000 \mathrm{~km} \mathrm{~s}^{-1}$ peak begins to dominate and its two component nature becomes more clear. Moreover, there is a continuing fading of the inner peaks to the point where they disappear into the broad emission by day 672/698. As previously mentioned, by this last epoch, the broad emission is a combination of the light echo spectrum and the weak residual ejecta emission. However, the residual flux beyond $5000 \mathrm{~km} \mathrm{~s}^{-1}$ is unlikely to originate from either of these two sources and remains unexplained. There is also an indication that the radial velocities of both sets of components get smaller with time. This

\footnotetext{
$8 i=90^{\circ}$ is edge-on
}

Table 4

Blackbody Fits

\begin{tabular}{lcccccccc}
\hline \hline \multirow{2}{*}{$\begin{array}{l}\text { Epoch } \\
\text { days })\end{array}$} & \multicolumn{3}{c}{ Blackbody Optical } & & \multicolumn{3}{c}{ Mod. Blackbody IR } & \multirow{2}{*}{$L_{\text {tot }}\left(L_{\odot}\right)$} \\
\cline { 2 - 4 } & $T(\mathrm{~K})$ & $R(\mathrm{AU})$ & $L\left(L_{\odot}\right)$ & & $T(\mathrm{~K})$ & $R(\mathrm{AU})$ & $L\left(L_{\odot}\right)$ & \\
\hline 300 & 5100 & 5.7 & $9.2 \mathrm{e} 5$ & & 580 & 1824 & $2.4 \mathrm{e} 6$ & $3.3 \mathrm{e} 6$ \\
455 & 5100 & 2.9 & $2.3 \mathrm{e} 5$ & & 490 & 1860 & $1.1 \mathrm{e} 6$ & $1.3 \mathrm{e} 6$ \\
667 & 5100 & 1.6 & $7.5 \mathrm{e} 4$ & & 600 & 767 & $5.1 \mathrm{e} 5$ & $5.8 \mathrm{e} 5$ \\
\hline
\end{tabular}

is similar to the behavior of the blue component of SN $1998 \mathrm{~S}$ which moved to smaller velocities from day 249 to day 658 , and was explained as an interaction of the shock with more massive sections of CSM (Pozzo et al. 2004).

\subsection{Radiative Transfer Modeling}

The SEDs of SN 2007od shown in Figure 5 all show strong IR excesses attributable to warm dust. The SED at 300 days can be fitted well by the sum of a blackbody with a temperature of $5100 \mathrm{~K}$ representing hot, optically-thick gas in the ejecta and a modified blackbody with a temperature of $580 \mathrm{~K}$ subject to a $\lambda^{-1}$ emissivity law representing the dust. A similar fit to the day 455 SED uses a $5100 \mathrm{~K}$ blackbody and a $490 \mathrm{~K}$ modified blackbody, and for day 667 a $5100 \mathrm{~K}$ blackbody and a $600 \mathrm{~K}$ modified blackbody. The fits are shown in Figure 5 and the relevant parameters are compiled in Table 4. The IR emission could arise from pre-existing dust in the CSM and/or new dust condensing in either or both of the CDS and the ejecta itself. IR echoes from pre-existing CSM dust heated by the earlytime luminosity are often seen in other SNe. The position of the dust in SN 2007od cannot be inferred from IR observations alone, but when combined with the evidence provided by the asymmetry in the $\mathrm{H} \alpha$ emission components, it suggests that the IR emission is coming from dust forming in the ejecta or the CDS, and not from an IR echo. The very early onset of dust formation (between 114 and 232 days) further suggests that the dust formation is occurring in the CDS since the ejecta at this time would still be too warm for dust condensation (Kozasa et al. 1991).

In order to better understand the amount and composition of the dust forming within SN 2007od, we used our 3D Monte Carlo radiative transfer (RT) code MOCASSIN (Ercolano et al. 2005, and references therein). For the purposes of our RT models, we will assume that the dust and source luminosity is spread throughout a spherical, expanding shell with an inner radius, $R_{\text {in }}$, and an outer radius, $R_{\text {out }}$. The luminosity at each location is proportional to the local density. With this simple model, we can quantify the total amount of dust in the system as a whole (ejecta + CDS) and monitor its variation from day 300 to 667 . We have modeled two different dust distributions. Following radiative transfer models of other Type II SNe (Sugerman et al. 2006; Ercolano et al. 2007), the "smooth" model for SN 2007od has the dust uniformly distributed throughout the shell according to an $r^{-2}$ density profile, while the "clumpy" model reflects an inhomogeneous distribution of spherical clumps embedded within an interclump medium of density $\rho$. The irradiating photons are only produced in the interclump medium, while the clumps are assumed to be dark (see Ercolano et al. 2007 for further discussion of this issue). We have adopted a standard grain size distribution of $a^{-3.5}$ between 0.005 and $0.05 \mu \mathrm{m}$ (Sugerman et al. 2006; Meikle et al. 2007; Kotak et al. 2009) and have tested two different concentrations, i.e., a dust model dominated by silicates $(75 \% \mathrm{Si}$; $25 \% \mathrm{AC})$ and one dominated by amorphous carbon (75\% AC; 
Table 5

Monte Carlo Radiative Transfer Models

\begin{tabular}{lcccccccc}
\hline \hline Epoch & $\mathrm{AC} / \mathrm{Si}$ & \multicolumn{4}{c}{ Smooth } & \multicolumn{2}{c}{ Clumpy } \\
\cline { 2 - 8 } (days) & & $T(\mathrm{~K})$ & $R_{\text {in }}(\mathrm{AU})$ & $R_{\text {out }}(\mathrm{AU})$ & $L_{\text {tot. }}\left(L_{\odot}\right)$ & $\tau_{v}$ & $M_{d}\left(M_{\odot}\right)$ & $M_{d}\left(M_{\odot}\right)$ \\
\hline 300 & $0.75 / 0.25$ & 6000 & 50.1 & 2005 & $3.4 \mathrm{e} 6$ & 1.50 & $1.7 \mathrm{e}-4$ & $2.5 \mathrm{e}-4$ \\
455 & $0.75 / 0.25$ & 6000 & 50.1 & 1537 & $1.4 \mathrm{e} 6$ & 2.00 & $1.9 \mathrm{e}-4$ & $4.2 \mathrm{e}-4$ \\
667 & $0.75 / 0.25$ & 6000 & 50.1 & 936 & $8.1 \mathrm{e} 5$ & 2.75 & $1.8 \mathrm{e}-4$ & $4.2 \mathrm{e}-4$ \\
\hline
\end{tabular}

$25 \% \mathrm{Si})$. For each of these models, we used the optical grain constants of Hanner (1988) and Draine \& Lee (1984), respectively.

The main input parameters for the model are the ejecta temperature and luminosity, the inner and outer radii of the shell, and the mass of dust present. Initial values of the luminosity, temperature, and approximate size of the dust shell are based on the results of our blackbody analysis above. Tuning the dust mass, along with slight variations in the other parameters, allowed us to obtain a good fit to the optical and Spitzer/IRAC points for the first two epochs. We should point out that without the 5.8 and $8.0 \mu \mathrm{m}$ data on day 667 , we were unable to make reliable constraints on the dust mass at this epoch. The best fit to the data was obtained with a smooth dust distribution and is shown in Figure 5, and the corresponding input parameters are listed in Table 5. This table also lists the dust mass calculated by our clumpy model.

Although no attempts were made to fine tune the models to match the 16 and $24 \mu \mathrm{m}$ flux upper limits on day $\sim 455$, care was taken to ensure that our preferred model did not predict emitted fluxes that would have been detected given our experimental setup. We found that we were unable to account for this relatively low flux at 16 and $24 \mu \mathrm{m}$ with compositions dominated by silicate dust (see Figure 5). All other parameters being equal, transitioning from amorphous carbon dust to silicate dust has the effect of broadening the IR peak as the SED will then be increased by strong silicate emission features at 10 and $18 \mu \mathrm{m}$. These two emission bumps are clearly seen in the Si model in Figure 5. The conclusion is that the silicate-dominated model predicts too much flux beyond $10 \mu \mathrm{m}$ at day 455 leading us to favor the amorphous-carbon-dominated model with $75 \% \mathrm{AC}$ and $25 \%$ Si. Given this concentration, our best smooth model predicts a total of $1.7 \times 10^{-4} M_{\odot}$ and $1.9 \times 10^{-4} M_{\odot}$ of dust on days 300 and 455 , respectively, with an increase in $\tau_{v}$ from 1.5 to 2.0 over the two epochs. This implies a total of $2 \times 10^{-5} M_{\odot}$ of new dust forming between day 300 and 455 after explosion. The values of $\tau$ at $6600 \AA$ predicted by our MOCASSIN fits were 1.3 and 1.8 for days 300 and 460, respectively. These opacities are adequate to explain the inner peak height ratios discussed in Section 3.2. Although the constraints were less reliable on the dust mass at day 667, we were able to show that the available data is consistent with our previous epochs with a predicted dust mass of $1.8 \times 10^{-4} M_{\odot}$ and $\tau_{v}=2.75$. The best clumpy model gave a total dust mass of $2.5 \times 10^{-4} M_{\odot}$ and $4.2 \times 10^{-4} M_{\odot}$ on days 300 and $455 / 667$, respectively, implying the formation of $1.7 \times 10^{-4} M_{\odot}$ of new dust formed. In each case, the quantity of dust formed is several orders of magnitude lower than that required to contribute significantly to the dust budget of the early universe (Morgan \& Edmunds 2003).

\section{SUMMARY}

SN 2007od is a very unusual Type IIP SN that shows strong evidence for early-time ( $\lesssim 232$ days) dust formation in the CDS created by the interaction between the ejecta and the CSM. Observations prior to day 82 for the optical light curve and prior to day 114 for the spectral evolution indicate that SN 2007od was a normal Type IIP SN. Photometry and spectra obtained on day 232 revealed that a large post-plateau luminosity drop $(\sim 4.5 \mathrm{mag})$ and the development of multi-peaked $\mathrm{H} \alpha$ emission had occurred during the period the SN was unobservable. The sharp luminosity decline between days 82 and 232 may have been caused by a low mass $\left(\leqslant 3 \times 10^{-3} M_{\odot}\right)$ of ${ }^{56} \mathrm{Ni}$ in the ejecta combined with the formation of new dust in the CDS. The presence of dust is supported by the red/blue asymmetries seen in the $\mathrm{H} \alpha$ components as well as the presence of a large IR excess. In SN 2007od, the dust formed between days 114 and 232 which is much earlier than seen for other Type IIP $\mathrm{SNe}$, but in line with $\mathrm{SNe}$ that are interacting with the CSM such as SN 1998S. There is also an indication in the observed flattening of the late-time light curve that a light echo from surrounding circumstellar or interstellar medium is present around SN 2007od. Flux from this echo also would make a significant contribution to the $\mathrm{H} \alpha$ emission profile at late times.

SN 2007od is unusual in that, while it is classified as a Type IIP, there is evidence for CSM lying close to the progenitor, even though it showed neither a narrow $\mathrm{H} \alpha$ component nor $\mathrm{X}$-ray emission which are both present in typical Type IIn SNe. We believe that the multi-peaked $\mathrm{H} \alpha$ emission in SN 2007od arises from a similar mechanism to that seen in SN 1998S and is consistent with the interaction of the ejecta with a circumstellar torus and blob. Assuming that scenario, the $\pm 1500 \mathrm{~km} \mathrm{~s}^{-1}$ emission arises from the ejecta interacting with a circumstellar torus, and the $-5000 \mathrm{~km} \mathrm{~s}^{-1}$ arises from a single cloud or blob of shocked CSM out of the plane of the CSM. The use of a torus and blob is to illustrate the ways that the multicomponent $\mathrm{H} \alpha$ emission may arise but should not be construed as the "true" geometry of the SN 2007od which is not well constrained by the data.

Our radiative transfer models have indicated that up to $4.2 \times 10^{-4} M_{\odot}$ of dust has formed in the CDS of SN 2007od. The models indicate that $2.5 \times 10^{-4} M_{\odot}$ was present on day 300 and an additional $1.7 \times 10^{-4} M_{\odot}$ formed by day 455 . This amount of new dust is similar to estimates for other CCSNe. For example, SN 1987A produced $7.5 \times 10^{-4} M_{\odot}$ of dust (Ercolano et al. 2007) and SN 2006jc an estimated $3 \times 10^{-4} M_{\odot}$ of dust (Mattila et al. 2008). Dust estimates for SN $2003 \mathrm{gd}$ currently yield values between $2 \times 10^{-4} M_{\odot}$ and $2 \times 10^{-2} M_{\odot}$ from Sugerman et al. (2006) and $4 \times 10^{-5} M_{\odot}$ from Meikle et al. (2007), both based on the same data. There seems to be a significant variation in the amount of dust produced from SN to SN, but none of them approach the $\sim 1 M_{\odot}$ of dust per SN needed to account for the massive amounts of dust seen in early-time galaxies at high redshift (Kozasa et al. 1989; Todini \& Ferrara 2001). Therefore, in the few dust-forming Type II SNe that have been observed, there is no evidence that these objects are major contributors to the dust-rich early galaxies seen at high redshift. The sample is still very meager, and more such SNe need to be observed with 
Table 6

Tertiary BVRI Standards for UGC 12846

\begin{tabular}{lcccc}
\hline \hline Star & $B$ & $V$ & $R$ & $I$ \\
\hline A & $16.989 \pm 0.014$ & $15.938 \pm 0.011$ & $15.338 \pm 0.006$ & $14.820 \pm 0.017$ \\
B & $17.480 \pm 0.020$ & $16.357 \pm 0.011$ & $15.720 \pm 0.006$ & $15.153 \pm 0.018$ \\
C & $17.266 \pm 0.015$ & $16.109 \pm 0.011$ & $15.403 \pm 0.006$ & $14.786 \pm 0.017$ \\
D & $17.966 \pm 0.019$ & $17.133 \pm 0.014$ & $16.672 \pm 0.010$ & $16.343 \pm 0.068$ \\
E & $18.146 \pm 0.021$ & $17.240 \pm 0.014$ & $16.708 \pm 0.010$ & $16.267 \pm 0.039$ \\
\hline
\end{tabular}

good temporal and wavelength coverage. We are still continuing the monitoring of SN 2007od in the optical and IR.

We thank the anonymous referees for valuable suggestions which have improved this paper. We also thank Stéphane Blondin, Robert Kirshner, and the rest of the CfA SN group for providing the early-time spectra of SN 2007od and to Ryan Chornock at Berkeley for allowing us access to his early time light curve and spectroscopic data. This work has been supported by NSF grant AST-0707691 and NASA GSRP grant NNX08AV36H. This work is based in part on observations made with the Spitzer Space Telescope, which is operated by the Jet Propulsion Laboratory, California Institute of Technology under a contract with NASA. Support for this work was provided by NASA through an award (RSA No. 1346842) issued by JPL/Caltech. A portion of these data was obtained at the Gemini Observatory, which is operated by the Association of Universities for Research in Astronomy (AURA) under a cooperative agreement with the NSF on behalf of the Gemini partnership. The standard data acquisition has been supported by NSF grants AST-0503871 and AST-0803158 to A. U. Landolt.

\section{APPENDIX}

\section{TERTIARY STANDARDS FOR UGC 12846}

The photometric sequence of tertiary standard stars used for both SMARTS and Gemini photometry (Table 6) was obtained using the Y4KCam CCD on the Yale $1.0 \mathrm{~m}$ telescope operated at the Cerro Tololo Inter-American Observatory on the night of 2008 October 28 . The Y4KCam offered a field of view of $\sim 20^{\prime} \times$ $20^{\prime}$ and employed a set of standard Johnson-Kron-Cousins broadband BVRI filters. The processing of all images to remove instrumental signatures was accomplished using the standard techniques of subtracting a median filtered bias frame and dividing by master twilight flat field images for each filter. Also, the fringing effects in the $I$-band exposures were removed by scaling and subtracting a master fringe frame from the program images. In order to transform the instrumental magnitudes to the standard system, we observed between 30 and 35 different stars (depending on filter) from the lists of Landolt (2009) in addition to the SN 2007od field. Importantly, these stars were selected due to their ample range in color, and they were observed both near zenith and at a high airmass $(\sim 1.75)$. Thus, they provided a viable sample of stars to derive accurate color and extinction coefficients as well as magnitude zero points for each filter. The resulting rms scatter of the residuals from the transformations revealed that the photometry for selected stars in the SN 2007od field derived from this night's observations is accurate on the $1 \%-2 \%$ level depending on filter.

\section{REFERENCES}

Barbon, R., Benetti, S., Rosino, L., Cappellaro, E., \& Turatto, M. 1990, A\&A, 237, 79

Bertoldi, F., Carilli, C. L., Cox, P., Fan, X., Strauss, M. A., Beelen, A., Omont, A., \& Zylka, R. 2003, A\&A, 406, L55

Blondin, S., \& Calkins, M. 2007, Central Bureau Electronic Telegrams, 1119, 1 Chevalier, R. A., \& Fransson, C. 1994, ApJ, 420, 268

Couderc, P. 1939, Ann. d'Astrophys., 2, 271

Draine, B. T., \& Lee, H. M. 1984, ApJ, 285, 89

Dwek, E. 1998, ApJ, 501, 643

Elmhamdi, A., Chugai, N. N., \& Danziger, I. J. 2003a, A\&A, 404, 1077

Elmhamdi, A., et al. 2003b, MNRAS, 338, 939

Ercolano, B., Barlow, M. J., \& Storey, P. J. 2005, MNRAS, 362, 1038

Ercolano, B., Barlow, M. J., \& Sugerman, B. E. K. 2007, MNRAS, 375, 753

Fox, O., et al. 2009, ApJ, 691, 650

Fransson, C., et al. 2005, ApJ, 622, 991

Fukugita, M., Ichikawa, T., Gunn, J. E., Doi, M., Shimasaku, K., \& Schneider, D. P. 1996, AJ, 111, 1748

Gerardy, C. L., Fesen, R. A., Höflich, P., \& Wheeler, J. C. 2000, AJ, 119, 2968

Hanner, M. 1988, in Infrared Observations of Comets Halley and Wilson and Properties of the Grains (NASA C-3004; Washington, DC: NASA), 22

Immler, S., \& Brown, P. J. 2007, ATel, 1259, 1

Immler, S., Brown, P. J., Filippenko, A. V., \& Pooley, D. 2007a, ATel, 1290, 1

Immler, S., Pooley, D., Brown, P. J., Li, W., \& Filippenko, A. V. 2007b, ATel, 1284,1

Kasen, D., \& Woosley, S. E. 2009, ApJ, 703, 2205

Kotak, R., et al. 2009, ApJ, 704, 306

Kozasa, T., Hasegawa, H., \& Nomoto, K. 1989, ApJ, 344, 325

Kozasa, T., Hasegawa, H., \& Nomoto, K. 1991, A\&A, 249, 474

Landolt, A. U. 2009, AJ, 137, 4186

Leonard, D. C., Filippenko, A. V., Barth, A. J., \& Matheson, T. 2000, ApJ, 536, 239

Lucy, L. B., Danziger, I. J., Gouiffes, C., \& Bouchet, P. 1989, in Lecture Notes in Physics, Vol. 350, IAU Coll. 120: Structure and Dynamics of the Interstellar Medium, ed. G. Tenorio-Tagle, M. Moles, \& J. Melnick (Berlin: Springer), 164

Mattila, S., et al. 2008, MNRAS, 389, 141

Meikle, W. P. S., et al. 2007, ApJ, 665, 608

Mikuz, H., \& Maticic, S. 2007, Central Bureau Electronic Telegrams, 1116, 1

Morgan, H. L., \& Edmunds, M. G. 2003, MNRAS, 343, 427

Pastorello, A., et al. 2004, MNRAS, 347, 74

Patat, F. 2005, MNRAS, 357, 1161

Patat, F., Benetti, S., Cappellaro, E., \& Turatto, M. 2006, MNRAS, 369, 1949 Pooley, D., et al. 2002, ApJ, 572, 932

Pozzo, M., Meikle, W. P. S., Fassia, A., Geballe, T., Lundqvist, P., Chugai, N. N., \& Sollerman, J. 2004, MNRAS, 352, 457

Sahu, D. K., Anupama, G. C., Srividya, S., \& Muneer, S. 2006, MNRAS, 372 , 1315

Sirianni, M., et al. 2005, PASP, 117, 1049

Smith, N., Foley, R. J., \& Filippenko, A. V. 2008, ApJ, 680, 568

Smith, N., et al. 2009, ApJ, 695, 1334

Sollerman, J., Cumming, R. J., \& Lundqvist, P. 1998, ApJ, 493, 933

Sugerman, B. E. K. 2003, AJ, 126, 1939

Sugerman, B. E. K., et al. 2006, Science, 313, 196

Todini, P., \& Ferrara, A. 2001, MNRAS, 325, 726

Welch, D. L., Clayton, G. C., Campbell, A., Barlow, M. J., Sugerman, B. E. K., Meixner, M., \& Bank, S. H. R. 2007, ApJ, 669, 525

Witt, A. N., \& Gordon, K. D. 1996, ApJ, 463, 681

Wooden, D. H., Rank, D. M., Bregman, J. D., Witteborn, F. C., Tielens, A. G. G. M., Cohen, M., Pinto, P. A., \& Axelrod, T. S. 1993, ApJS, 88, 477

Woosley, S. E., \& Weaver, T. A. 1986, ARA\&A, 24, 205 